\title{
NONLINEAR PROPAGATION
}

$O F$

LOWER HYBRID WAVES IN A PLASMA

BY

$P, K, K A W, C, Z$, CHENG

AND

LIU CHEN

PLASMA PHYSICS LABORATORY

\section{DISTRIBUTION OF THIS DOCUMENT IS UNLIMITED}

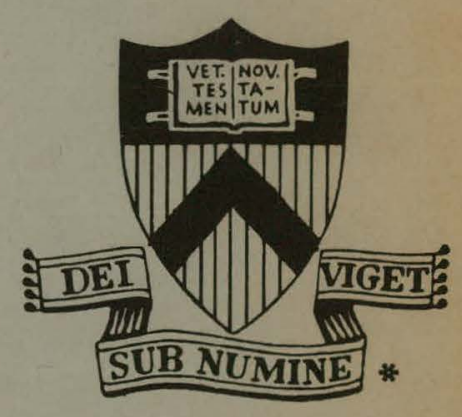

\section{PRINCETON \\ UNIVERSITY PRINCETON, NEW JERSEY}

This work was supported by U. S. Energy Research and Development Administration Contract E(11-1)-3073. Reproduction, translation, publication, use and disposal, in whole or in part, by or for the United States Government is permitted. 


\section{DISCLAIMER}

This report was prepared as an account of work sponsored by an agency of the United States Government. Neither the United States Government nor any agency Thereof, nor any of their employees, makes any warranty, express or implied, or assumes any legal liability or responsibility for the accuracy, completeness, or usefulness of any information, apparatus, product, or process disclosed, or represents that its use would not infringe privately owned rights. Reference herein to any specific commercial product, process, or service by trade name, trademark, manufacturer, or otherwise does not necessarily constitute or imply its endorsement, recommendation, or favoring by the United States Government or any agency thereof. The views and opinions of authors expressed herein do not necessarily state or reflect those of the United States Government or any agency thereof. 


\section{DISCLAIMER}

Portions of this document may be illegible in electronic image products. Images are produced from the best available original document. 
This report was prepared as an account of work sponsored by the United States Government. Neither the United States nor the United States Energy Research and Development Administration, nor any of their employees, nor any of their contractors, subcontractors, or their employees, makes any warranty, express or implied, or assumes any legal liability or responsibility for the accuracy, completeness or usefulness of any information, apparatus, product or process disclosed, or represents that its use would not infringe privately owned rights.

Printed in the United States of America.

Available from

National Technical Information Service

U. S. Department of Commerce 5285 Port Royal Road

Springfield, Virginia 22151

Price: Printed Copy \$_*; Microfiche $\$ 3.00$

*Pages

$1-50$

51-150

$151-325$

326-500

501-1000
NTIS

Selling Price

$\$ 4.00$

5.45

7.60

10.60

13.60 
Nonlinear Propagation of Lower Hybrid Waves in a Plasma

P. K. Kaw, C. Z. Cheng, and Liu Chen

Plasma Physics Laboratory, Princeton University

Princeton, New Jersey 08540 
Nonlinear Propagation of Lower Hybrid Waves in a Plasma P. K. Kaw, C. Z. Cheng, and Liu Chen Princeton University, Plasma Physics Laboratory Princeton, N. J. 08540 USA

\begin{abstract}
In this paper we use a model equation to investigate the competition between the electron $\underset{\mathrm{m}}{\mathrm{E}} \times \underset{\mathrm{B}}{\mathrm{B}}$ nonlinearities (which lead to parametric instabilities and anomalous absorption) and the electron polarization drift and ion nonlinearities (which induce filamentation and self-focusing effects) in the propagation of a large amplitude lower hybrid wave in a plasma. Our conclusion is that the former dominates for plasma regions with $\omega_{\mathrm{pi}}^{2}>\omega_{\mathrm{ci}}^{2}$ (i. e. , everywhere except in a thin skin region).
\end{abstract}




\section{INTRODUCTION}

One of the major contenders for the auxiliary heating of tokamak plasmas with radio-frequency fields is the use of lower hybrid resonance frequencies [1]. Extensive experimental [2,3] and theoretical [4-6] work in this area has been done in recent years. One crucial problem in this heating scheme (as with others) is the propagation of rf energy into the plaoma interiur. Linear theories of lower hybrid wave propagation in weakly non-uniform plasmas have been studied by many anthors $\left[\begin{array}{lll}1, & 1 & 6\end{array}\right]$.

Recently, Morales and Lee [7] have studied certain nonlinear effects in lower hybrid wave propagation. They restrict their attention to a twodimensional situation (viz. the plane containing dc magnetic field and the oscillating electric field of the wave) and consider wave amplitudes large enough to produce significant density cavities due to the component of ponderomotive force along the lines of force. These density cavities then modify the usual wave propagation by distorting the linear lower hybrid cones. The resultant self-consistent description of nonlinear distortion of lower hybrid cones is then shown to be governed by a modified Korteweg-de Vries equation. This equation is known to admit multiple-soliton solutions. Morales and Lee [7] thus conclude that nonlinearly, lower hybrid waves will distort and take the form of filaments with intense localized electric fields, which may produce strong plasma heating either in the surface or in the interior, depending on the various plasma parameters. 
In this paper, we point out that by restricting their attention to a two-dimensional problem, Morales and Lee [7] have missed the dominant nonlinear effect for lower hybrid waves. The ponderomotive force nonlinearities in their calculation enter through the polarization drift and parallel motion of electrons and produce a significant effect when $|E|^{2} / 8 \pi \mathrm{n} T \lesssim 0(1)$. The dominant nonlinear effect (ignored by Morales and Lee [7]) arises from the $\underset{m}{E} \times \underset{m}{B}$ motion of electrons, which parametrically couples energy from the given lower hybrid wave into other decay waves propagating in the third dimension viz., normal to $\underset{m}{E}$ and $\underset{m_{0}}{B}$. These parametric processes have been extensively studied recently $[8-10]$ and produce significant effects already when $c|\underset{m}{E}| / B_{m_{O}} c_{s} s 0(1)$, where $c_{S}$ and $c$ are respectively the ion-acoustic and light speeds. Comparing the two nonlinearities we find that $\underset{m}{E} \times \underset{m o}{B}$ nonlinearities dominate over the ponderomotive force nonlinearity by a factor $\left.\sim\left(\mathrm{k}_{\perp} \lambda_{\mathrm{Di}}\right) \omega_{\mathrm{pi}}^{2} / \omega_{\mathrm{ci}}^{2}\right)\left[z_{0}\left(\mathrm{~m}_{\mathrm{i}} / \mathrm{m}_{\mathrm{e}}\right)^{1 / 2} \gg 1\right.$, everywhere except in a very thin skin near the plasma edge]. We, thus, conclude that, to be applicable to realistic plasma situations, the nonlinear propagation calculation of Morales and Lee [7] must include $\underset{\mathrm{m}}{\mathrm{E}} \times \underset{\mathrm{m}}{\mathrm{B}}$ coupling effects. This is what we attempt to do below.

A detailed calculation of the $\underset{m}{E} \times \underset{m}{\mathrm{~B}}$ nonlinear coupling involves the study of parametric excitation of decay waves, their nonlinear saturation 1 and reaction back on the pump in the form of pump depletion etc. Such a calculation is quite involved and is currently in progress. One can, however, investigate the effect of $\underset{m}{E} \times \underset{m o}{B}$ coupling terms on the two-dimensional nonlinear propagation by a simple model calculation. Physically, eneryy 
deposition into parametrically excited decay waves, propagating in the third $(\underset{m}{E} \times \underset{m}{B})$ dimension can be simulated by a model nonlinear absorption coefficient, which is then incorporated into the two-dimensional nonlinear equation. We present results of such a model calculation below. Our basic result is that inclusion of the pump depletion term due to $\underset{\mathrm{m}}{\mathrm{E}} \times \mathrm{B}$ o coupling, almost completely suppresses the filamentation effect for most realistic parameters.

\section{MODEL EQUATION}

We shall now derive the model nonlinear equation for lower hybrid waves. Since we treat these waves in the electrostatic approximation, they obey the charge continuity equation

$$
\frac{\partial}{\partial t}(\nabla \cdot E)+4 \pi \nabla \cdot J=0
$$

where $\mathrm{J}$ is the total current density defined by

$$
J=e\left(n_{i m i} v_{m}-n_{e_{e}}^{v}\right)
$$

Nonlinear interaction of lower hybrid waves with each other generates low-frequency density fluctuations (such as through ponderomotive force effects, $\underset{m}{E} \times \underset{m_{0}}{B}$ coupling, etc.). Thus, we may write $n_{i, e}=n_{o}+n_{i, e}^{s}+$ $\mathrm{n}_{\mathrm{i}, \mathrm{e}}^{\mathrm{f}}$ where the quasi-neutral density fluctuations $\mathrm{n}_{i}^{\mathrm{s}} \approx \mathrm{n}_{\mathrm{e}}^{\mathrm{s}} \simeq \mathrm{n}_{\mathrm{s}}$ correspond to the low-frequency response and $n_{i, e}^{f}$ denotes the fast response at the lower hybrid frequency. In our further discussion below, we shall approximate the low-frequency response by a static-response assumption. Strictly 
speaking, this assumption includes only those parametric processes, which excite decay waves with frequency deviation from the pump, $\Delta \omega \ll \mathrm{kv}_{\text {ith }}$. However, we note that the new nonlinear coupling terms in this approximation have the correct order of magnitude even for parametric decays with $\Delta \omega \geq \mathrm{kv}_{\text {ith }}$. The assumption is thus appropriate even generally in the spirit of a model nonlinear calculation:

Combining Eqs. (1) and (2), we may now write the approximate highfrequency equation

$$
\nabla \cdot \ddot{m}+4 \pi e\left(n_{0}+n_{s}\right) \nabla \cdot\left(\dot{v}_{m i}-\dot{v}_{m e}\right)+4 \pi e\left(\dot{v}_{m i}-\dot{v}_{m e}\right) \cdot \nabla n s=0,
$$

where the dot denotes a' time derivative. Equation (3) ignores terms proportional to the low-frequency $\Delta \omega$, in the spirit discussed above. It is well known that for lower hybrid frequencies, ions behave as unmagnetized species and electrons as highly magnetized ones. The cold plasma response of the ions and electrons to the high-frequency fields is given by

$$
\begin{aligned}
& \dot{\mathrm{v}}_{\mathrm{m} \mathrm{i}}=\mathrm{eE} / \mathrm{m}_{\mathrm{i}}, \\
& \dot{v}_{m e}=c(\underset{m}{E} \times \hat{z}) / B_{0}-\left(m_{e} c^{2} / e B_{0}^{2}\right) \underset{m}{\ddot{E}}-(e / m) E_{z} \hat{z} .
\end{aligned}
$$

The electron response consists of an $\underset{m}{E} \times \underset{m o}{B}$ drift, a polarization drift and a velocity parallel to the $d c$ magnetic field $\underset{m_{0}}{B_{0}}=B_{0} \hat{z}$. Combining Eqs. (3) and (4) we may write 


$$
\begin{gathered}
\nabla \cdot\left\{\left[1+\frac{\omega_{p e}^{2}}{\omega_{c e}^{2}}\left(1+\frac{n_{s}}{n_{0}}\right)\right] \underset{m}{\ddot{E}}\right\}+\nabla \cdot\left[\omega_{p i}^{2}\left(1+\frac{n_{s}}{n_{o}}\right) \underset{m}{E}\right] \\
+\frac{\partial}{\partial z}\left[\omega_{p e}^{2}\left(1+\frac{n_{s}}{n_{o}}\right) E_{z}\right]=\frac{4 \pi e c n_{o}}{B_{o}}\left(\sum_{m}^{\dot{E}} \times \hat{z}\right) \cdot \nabla\left(\frac{n_{s}}{n_{0}}\right),
\end{gathered}
$$

where $\omega_{\text {pe }}$ and $\omega_{\text {ce }}$ are respectively the electron plasma and electron cyclotron frequencies, etc. This equation dnes not talce account of the thermal dispersive effects of lower hybrid waves. This effect can readily be included on the left side of Eq. (5), following standard methods $[7,11]$.

Our next task is to express the low-frequency density fluctuations $\mathrm{n}_{\mathrm{s}}$ in terms of the high-frequency fields. Since the low-frequency response is to be approximated by static response equations (see discussion above), the relevant equations may simply be obtained by balancing the rmal pressure gradients and self-consistent field forces with the ponderomotive force terms:

$$
\begin{aligned}
& 0 \simeq-\frac{T_{e}}{n_{e} m_{e}} \frac{\partial}{\partial z}\left(n_{e}\right)-\left(v_{e} \cdot \nabla\right) v_{e z}+\frac{e}{m_{e}} \frac{\partial}{\partial z} \phi_{s}, \\
& 0 \simeq-\frac{T_{i}}{n_{i} m_{i}} \frac{\partial}{\partial z}\left(n_{i}\right)-\left(v_{i} \cdot \nabla\right) v_{i z}-\frac{e}{m_{i}} \frac{\partial}{\partial z} \phi_{s} .
\end{aligned}
$$

Eliminating $\phi_{s}$ by the use of quasi-neutrality condition $n_{e} \simeq n_{i}, u s i n g$. Eqs. $(4 \mathrm{a}, \mathrm{b})$ to eliminate $\mathrm{v}_{\mathrm{i}}, \underset{\mathrm{m}}{\mathrm{v}}$, and taking the time-dependence of high-frequency fields $\underset{m}{E}(t)=\underset{m}{E} \exp (-i \omega t)+c . c$, we obtain 


$$
\begin{aligned}
\frac{n_{e}}{n_{0}} \equiv 1+\frac{n_{s}}{n_{0}}=\exp [ & -\frac{i c e}{2 \omega B_{0} T}\left(E_{y} E_{x}^{* *}-E_{x} E_{y}^{*}\right)+\frac{m_{e} c^{2}}{2 T B_{0}^{2}}\left(\left|E_{x}\right|^{2}+\left|E_{y}\right|^{2}\right) \\
& \left.-\frac{e^{2}}{2 m \omega^{2} T}\left|E_{z}\right|^{2}-\frac{e^{2}}{2 m_{i} \omega^{2} T}|E|^{2}\right]
\end{aligned}
$$

where $T=T_{e}+T_{i}$. Following Morales and Lee [7], the last three terms in the exponent may be combined by using the linearized dispersion relation. for lower hybrid wave and the exponential may be expanded to yield

$$
\frac{n_{s}}{n_{0}} \simeq-\frac{i c e}{2 \omega B_{0} T}\left(E_{y} E_{x}^{*}-E_{x} E_{y}^{*}\right)-\frac{|E|^{2}}{8 \pi n_{0} T}
$$

For further simplification we now assume that the pump is confined to the $x-z$ plane and that the decay waves are propagating in the $y$-direction. We may now split the low-frequency density as

$$
\mathrm{n}_{\mathrm{s}}=\mathrm{n}_{\mathrm{so}}+\mathrm{n}_{\mathrm{s} 1}(\Delta \omega)
$$

where

$$
\frac{\mathrm{n}_{\mathrm{so}}}{\mathrm{n}_{\mathrm{O}}} \simeq-\frac{\left|\mathrm{E}_{\mathrm{o}}\right|^{2}}{8 \pi \mathrm{n}_{\mathrm{o}} \mathrm{T}}
$$

is the density response due to the pump wave self-interaction and (since

$$
\begin{aligned}
& \left.E_{o y}=0\right) \\
& \qquad \frac{n_{s l}}{n_{o}}(\Delta \omega)=-\frac{i c e}{2 \omega B_{0} L^{\prime}}\left(E_{o y} E_{1 x}^{*}-E_{o x} E_{l y}^{*}\right)=\frac{i c e}{2\left(\alpha B_{0} T\right.} E_{o x} E_{l y}^{*}
\end{aligned}
$$


is the low-frequency density fluctuation generated by the pump wave $E_{0}(\omega)$ interacting with the decay. wave $E_{1}(\omega-\Delta \omega)$. We may now rewrite Eq. (5) as

$$
\begin{aligned}
& \nabla \cdot\left[1+\frac{\omega_{p e}^{2}}{\omega_{c e}^{2}}\left(1+\frac{n_{s o}}{n_{0}}\right) \underset{m_{0}}{\ddot{E}}\right]+\nabla \cdot\left[\omega_{p i}^{2}\left(1+\frac{n_{s o}}{n_{o}}\right) E_{0}\right] \\
& +\partial_{z}\left[\omega_{p e}^{2}\left(1+\frac{n_{s o}}{n_{o}}\right) E_{o z}\right]=\frac{4 \pi e c}{B_{0}} n_{o}\left(\dot{E}_{m i} \times \hat{z}\right) \cdot \nabla\left(\frac{n_{s}}{n_{0}}\right) .
\end{aligned}
$$

The right side thus corresponds to purmp depletion due to coupling to decay waves propagating in the $\mathrm{y}$-direction. We may approximate the magnitude of right side as

$$
\left.\left|\frac{4 \pi e c n_{0}}{B_{0}}(-i \omega) E_{i y} \frac{d}{d x} \frac{n_{s}}{n_{0}}\right| \simeq\left|\frac{4 \pi e^{2} c^{2} n_{o}}{2 T B_{0}^{2}}\right| E_{l y}\right|^{2} k_{\perp} E_{o x} \mid
$$

where $\left|k_{\perp}\right| \simeq|\partial / \partial x|$ is the typical perpendicular structure length of the lower hybrid wave. Using the results [12] of weak turbulence theory saturation studies of decay instabilities, we note that saturation of decay waves occurs roughly when $\left|\mathrm{E}_{1 \mathrm{y}}\right|^{2} \simeq \theta\left|\mathrm{E}_{\mathrm{o}}\right|^{2}$ where $\theta$ is a constant of order unity [12]. We may, thus, approximate the purmp depletion term as $\left.\left|\left(4 \pi e^{2} c^{2} n_{0} / 2 B_{0}^{2} T\right)\right| E_{o}\right|^{2} E_{o x}\left(\theta k_{1}\right) \mid$. The final model equation for nonlinear propagation of lower hybrid waves may be written as

$$
\begin{aligned}
& \dot{\nabla} \cdot\left[1+\frac{\omega_{p e}^{2}}{\omega_{c e}^{2}}\left(1-\frac{|E|^{2}}{8 \pi n_{0} T}\right) E\right]-\nabla \cdot\left[\frac{\omega_{p i}^{2}}{\omega^{2}}\left(1-\frac{|E|^{2}}{8 \pi n_{0} T}\right) E\right] \\
& -\frac{\partial}{\partial z}\left[\frac{\omega_{\mathrm{pe}}^{2}}{\omega^{2}}\left(1-\frac{|E|^{2}}{8 \pi n_{0} T}\right) E_{z}\right]+\left(\frac{4 \pi e c n_{0}}{\omega B_{0}}\right)^{2} k_{1} \frac{|E|^{2}}{8 \pi n_{0} T} E_{x}=0 \text {. }
\end{aligned}
$$


The problem may now be reduced to a two-dimensional one (in the $x-z$ plane) where the new term plays the role of an anomalous absorption term, taking account of deposition of energy into decay waves propagating into the third dimension. Introducing

$$
\begin{aligned}
& \bar{c}=\left(\frac{K_{\perp 0}}{\left|K_{\| 0}\right|}\right)^{1 / 2}, \quad K_{10}=1+\frac{\omega_{p e}^{2}}{\omega_{c e}^{2}}-\frac{\omega_{p i}^{2}}{\omega^{2}}, \quad K_{\| 0}=1-\frac{\omega_{p e}^{2}}{\omega^{2}}-\frac{\omega_{p i}^{2}}{\omega^{2}} \text {, } \\
& \xi=(\alpha / \delta)^{1 / 2}\left(\omega / v_{i}\right)(x-\bar{c} z), \quad \quad \tau=\left(\alpha^{3 / 2} / \delta^{1 / 2}\right)\left(\bar{c} z / K_{\perp 0}\right)\left(\omega / v_{i}\right) \text {, } \\
& \mathrm{u}=\mathrm{E}_{\mathrm{x}} /\left(8 \pi \mathrm{n}_{\mathrm{o}} \mathrm{T}\right)^{1 / 2}, \quad \quad \quad \beta=\mathrm{k}_{1} \lambda_{\mathrm{Di}}\left(\omega_{\mathrm{pi}}^{2} / \omega_{\mathrm{ci}}^{2}\right)\left(\delta^{1 / 2} / \alpha^{3 / 2}\right)\left(\omega_{\mathrm{pi}} / \omega\right) \theta \\
& \alpha=\frac{\omega_{p e}^{2}}{\omega_{c e}^{2}}-\frac{\omega_{p i}^{2}}{\omega^{2}}+\frac{K_{\perp 0}}{\left|K_{\| 0}\right|} \frac{\omega_{p e}^{2}}{\omega^{2}}, \quad \delta \simeq \frac{\omega_{p i}^{2}}{\omega^{2}} \text {, }
\end{aligned}
$$

and proceeding in a manner similar to Morales and Lee [7], one obtains

$$
\frac{\partial u}{\partial \tau}+u^{2} \frac{\partial u}{\partial \xi}+\beta u^{3}+\frac{\partial^{3} u}{\partial \xi^{3}}=0 .
$$

The last term is the standard thermal dispersive correction $[7,11]$ to lower hybrid waves. Note that although the exact form of the new term $\beta u^{3}$ is not accurate (note we approximated derivatives $\partial / \partial \mathbf{x}, \partial / \partial \mathbf{y}$, by $\left|k_{\perp}\right|$ ), both its magnitude (viz. magnitude of $\beta$ ) and physicall consequence (viz. anomalous absorption) are es sentially correct. 


\section{NUMERICAL RESULTS AND DISCUSSION}

We have studied Eq. (10) numerically for the initial condition

$$
\mathrm{u}(\xi, \tau=0)=3 /\left[1-0.1 \xi^{2}\right] \text {, }
$$

and three values of $\beta=0,0.1,0.5$. Note that we have chosen rather high amplitude of the pump wave $\left(E^{2} / 8 \pi n_{n} T \sim 9\right)$ and sharp initial $\xi$ structure ( a few ion Debye lengths), both features very favorable for filamentation. Note also that for conditions relevant to the center of a tokamak plasma, $\omega_{\mathrm{pe}} \sim \dot{\omega}_{\mathrm{ce}}$ and $\omega \sim \omega_{\mathrm{pi}}$ giving $\delta \sim \alpha \sim 1$ and $\beta \gg 1$. The condition $\beta \leq 1$ is therefore more relevant to the low-density plasma at the surface (where $\alpha \sim 1$ and $\mathrm{k}_{\perp} \lambda_{\mathrm{Di}} \sim 1, \quad \beta \sim\left(\mathrm{k}_{\perp} \mathrm{v}_{\mathrm{i}} / \omega\right)\left(\omega_{\mathrm{pi}}^{2} / \omega_{\mathrm{ci}}^{2}\right) \lesssim 1$ means $\omega_{p i} \sim\left(\omega \omega_{c i}^{2}\right)^{1 / 3} \sim(M / m)^{1 / 6} \omega_{c i}$. Figures $1(a, b)$ show that for negligible $\beta$, filamentation takes place as predicted by the calculation of Morales and Lee [4]. However, Fig. 1(c) shows that even for rather moderate values of $\beta$, filamentation is completely suppressed. The dominant feature of the nonlinear propagation is seen to be an anomalous absorption of the wave due to energy deposition into decay waves.

One can roughly estimate the value of $\beta$ at which the damping term plays the dominant role as follows. Consider the equation

$$
u_{\tau}+u^{2} u_{\xi}=0
$$

Clearly, a given $u(\xi)$ profile will steepen as a function of $\tau$ due to the nonlinear term. The typical steepening distance may be written as

$$
\tau_{\text {steep }} \sim\left(\xi_{\alpha} u_{o}^{2}\right)
$$


where $\xi_{0}$ is the original structure length. Similarly, for the equation

$$
u_{\tau}+\beta u^{3}=0
$$

there is an exact solution

$$
u=u_{0} /\left(1+\beta u_{0}^{2} \tau\right)
$$

showing that the wave amplitude decays with a typical damping length

$$
\tau_{\operatorname{damp}} \sim 1 / \beta \mathrm{u}_{\mathrm{o}}^{2}
$$

It is quite clear that damping effects will suppress and overwhelm the stecpening effects if

$$
\tau_{\text {damp }}<\tau_{\text {steep }} \quad \text { i. e.., } \beta \xi_{\mathrm{o}} \equiv \beta \mathrm{u}_{\mathrm{o}}^{2} /\left(\mathrm{du}_{\mathrm{o}}^{2} / \mathrm{d} \xi\right)>1
$$

Note that this condition is satisfied for case (c) shown in the Fig. 1 . Using Eq. (9) one can further show that this condition (for $\alpha \sim 1$, which is typical) may be written as $\omega_{\mathrm{pi}}^{2} / \omega_{\mathrm{ci}}^{2}>1$. The conclusion of this calculation is then that filamentation is likely to be masked and completely overwhelmed by the anomalous absorption due to $\mathrm{E} \times \mathrm{B}$ coupling induced parametric decay instabilities in most regions of an inhomogeneous confined plasma except in a very thin low density skin where $\omega_{\mathrm{pi}}^{2}<\omega_{\mathrm{ci}}^{2}$.

We have also repeated the calculation for the modified equation for an inhomogeneous plasma. Except for a WKB amplification of the pump wave amplitude, there was no qualitative change in the results.

What is the consequence of this anomalous absorption for lower hybrid wave penetration into the plasma? We can only speculate on the 
further fate of the decay waves produced by $\underset{m}{E} \times \underset{m}{B}$ coupling. If they are heavily damped, they may be absorbed at the surface and result in surface heating; on the other hand, if they are weakly damped, they can carry the energy to the plasma interior. It all depends on the details of the spectra of excited decay instabilities. A calculation investigating this effect is in progress.

\section{АGKNOW LEDGMENTS}

This work was supported by U. S. Energy Research and Development Administration Contract $\mathrm{E}(11-1)-3073$. 


\section{REFERENCES}

[1] STIX, T. H., Phys. Rev. Letters 15 (1965) 878.

[2] HOOKE, W. H. and BERNABEI, S., Phys. Rev. Letters $\underline{28}$ (1972) 407.

[3] HOOKE, W. H. and BERNABEI, S., Phys. Rev. Letters 29 (1972) 1218.

[4] BRIGGS, R. J. and PARKER, R. R., Phys. Rev. Letters 29 (1972) 852.

[5] FISHER, R. K. and GOULD, R. W., Phys. Fluids 14 (1971) 857.

[6] BELLAN, P. and PORKOLAB, M. , Phys. Fluids 17 (1974) 1592.

[7] MORALES, G. J. and LEE, Y. C., Phys. Rev. Letters 35 (1975)930。

[8] PORKOLAB, M., Phys. Fluids 17 (1974) 1432.

[9] KAW, P. K. in Advances in Plasma Physics, Eds. A. Simon and W. Thomson (Wiley 1976) Vol. 6, p, 179.

[10] KINDEL, J., OKUDA, H., and DAWSON, J., Phys. Rev. Letters $29(1972) 995$.

[11] KAW. P. K., Princeton Plasma Physics Laboratory Report MATT-1208 (1976).

[12] BERGER, R. L. and CHEN, Liu, Princeton Plasma Physics Laboratory Report MATT-1169 (1975). 


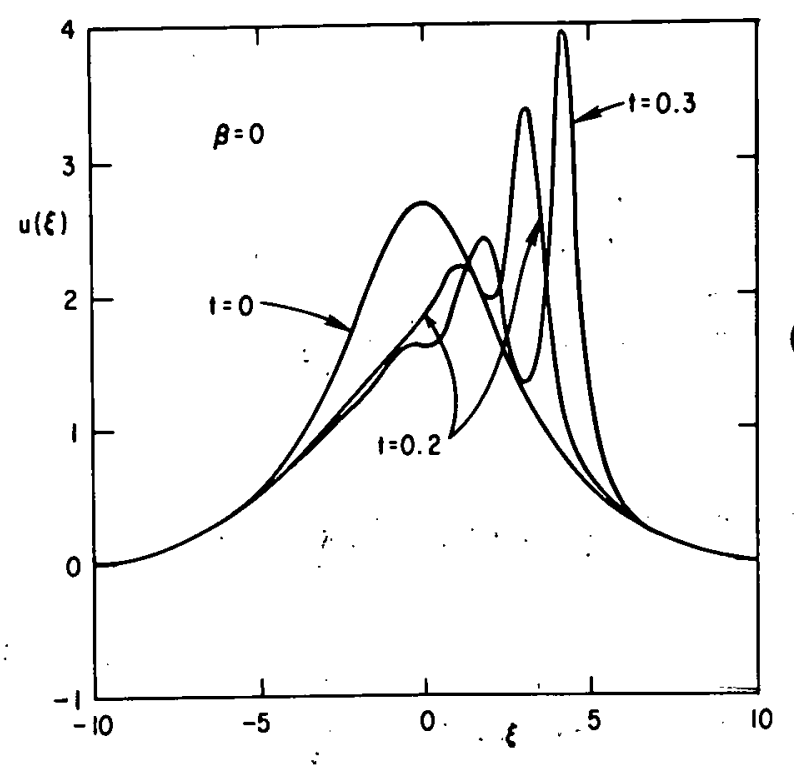

(a) $\beta=0 \quad \pi \widehat{0} 0$

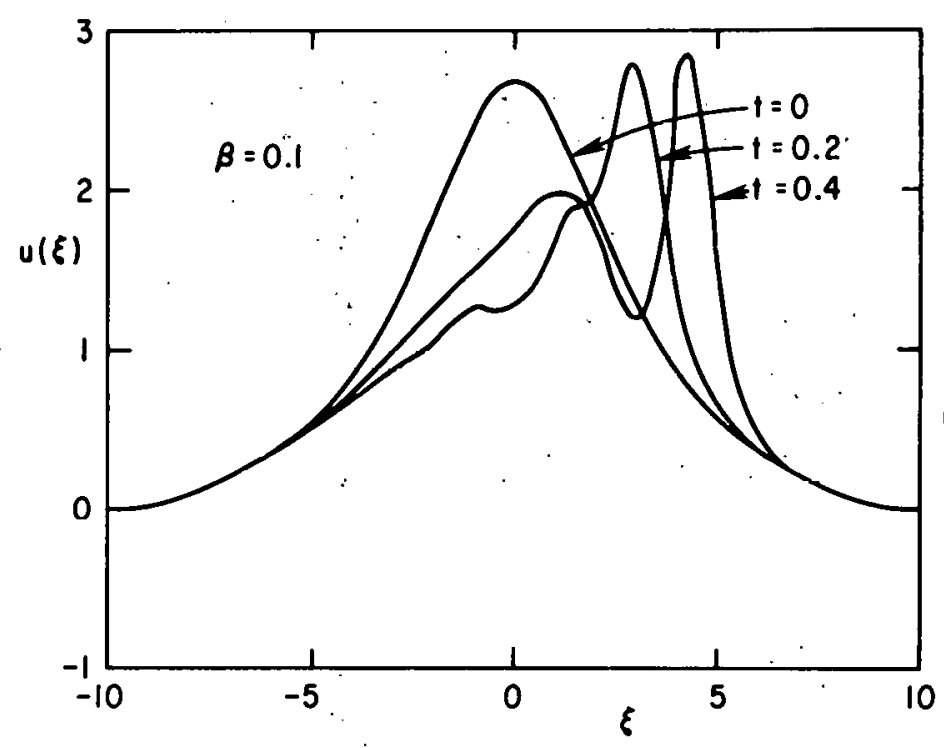

(b) $\beta=0.1$
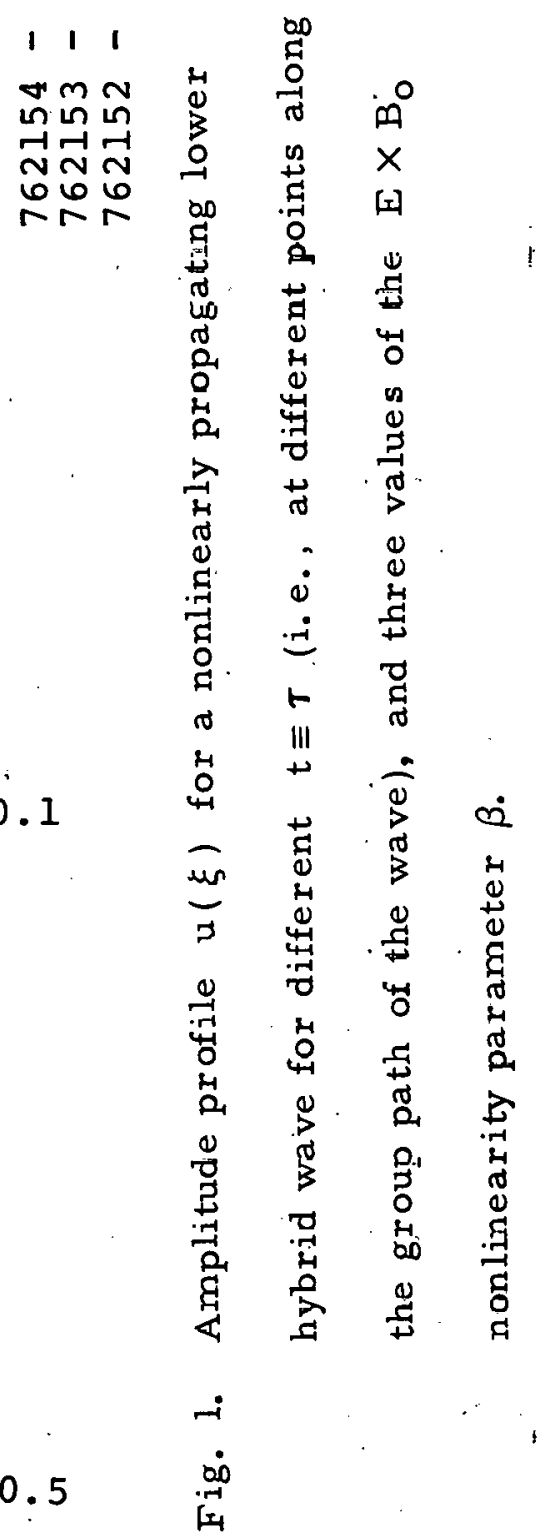